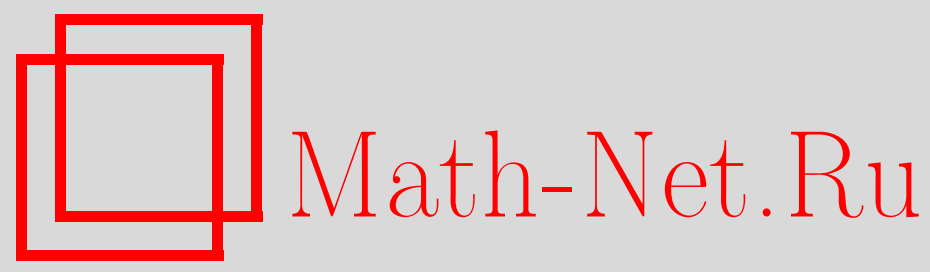

Л. И. Сазонов, Об асимптотическом поведении решения двумерной стационарной задачи обтекания вдали от обтекаемого тела, Матем. заметки, 1999, том 65, выпуск 2, 246 253

DOI: https://doi.org/10.4213/mzm1046

Использование Общероссийского математического портала Math-Net.Ru подразумевает, что вы прочитали и согласны с пользовательским соглашением http://www.mathnet.ru/rus/agreement

Параметры загрузки:

IP : 3.85 .73 .92

26 апреля 2023 г., $16: 24: 29$

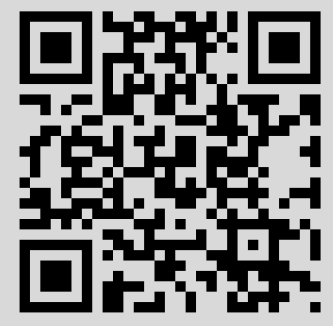




\section{ОБ АСИМПТОТИЧЕСКОМ ПОВЕДЕНИИ РЕШЕНИЯ ДВУМЕРНОЙ СТАЦИОНАРНОЙ ЗАДАЧИ ОБТЕКАНИЯ ВДАЛИ ОТ ОБТЕКАЕМОГО ТЕЛА}

\section{Л. И. Сазонов}

Для решения двумерной системы Навье-Стокса

$$
\Delta u-\nabla p=(u, \nabla) u, \quad \operatorname{div} u=0
$$

во внешней области $\Omega \subset \mathbb{R}^{2}$, имеющего конечньй интеграл Дирихле и удовлетворяющего условию $\lim _{|x| \rightarrow \infty} u(x)=(1,0)$, установлена оценка $|u(x)-(1,0)| \leqslant c|x|^{-\alpha}$, где $\alpha>1 / 4$. Из этой оценки следует асимптотика решения, свидетельствующая о наличии следа за обтекаемьм телом.

Библиограффия: 10 названий.

1. Пусть $\Omega$ - внешняя область в двумерной плоскости переменньх $x=\left(x_{1}, x_{2}\right)$ с достаточно гладкой границей $\partial \Omega$, не содержащая для определенности начала координат. Двумерная стационарная задача обтекания состоит в определении решения системы Навье-Стокса

$$
\nu \Delta w=\nabla p+(w, \nabla) w, \quad \operatorname{div} w=0
$$

в области $\Omega$, удовлетворяющего граничному условию

$$
\left.w\right|_{\partial \Omega}=0
$$

и предельному соотношению

$$
\lim _{|x| \rightarrow \infty} w(x)=w_{\infty} \quad\left(w_{\infty} \neq 0\right)
$$

Известно [1], что существует решение $\left(w_{L}, p_{L}\right)$ задачи $(1),(2)$, являющееся равномерным пределом на любой компактной области некоторой последовательности решений $\left(w_{R}, p_{R}\right)$ системы $(1)$ для усеченных областей $\Omega_{R}=\Omega \cap\{x:|x|<R\}$ с граничными условиями $\left.w_{R}\right|_{\partial \Omega}=0$ и $\left.w_{R}\right|_{|x|=R}=w_{\infty}$. Это решение удовлетворяет условию $\left|\nabla w_{L}\right| \in L_{2}(\Omega)$. В силу теорем о регулярности (см., например, [2]) любое обобщенное

Работа выполнена при поддержке Российского фонда фундаментальных исследований, грант № 96-01-01195. 
решение $(w, p)$ задачи $(1),(2)$ и, в частности, решение Лере $\left(w_{L}, p_{L}\right)$ является достаточно гладким в замькании области $\Omega$. Однако не доказано, что решение Лере является решением задачи обтекания, так как неизвестно принимает ли $w_{L}$ на бесконечности предписанное значение $w_{\infty}$; более того, в общем случае неизвестно даже существует ли $\lim _{|x| \rightarrow \infty} w_{L}$. Отметим, что при достаточно малой величине $\left|w_{\infty}\right| / \nu$ существование решения задачи обтекания установлено в работе [3].

Общие исследования поведения на бесконечности решений системы $(1),(2)$ были выполнены в работах [4]-[7]. Настоящая заметка непосредственно связана с [7], где для симметричного относительно оси $x_{1}$ решения задачи обтекания в симметричной области $\Omega$, удовлетворяющего соотношению $|\nabla w| \in L_{2}(\Omega)$, установлена оценка

$$
\left|w-w_{\infty}\right|=o\left(|x|^{-1 / 4-\varepsilon}\right) \text {, }
$$

где $\varepsilon>0$ достаточно мало.

Ниже этот результат установлен для общего (несимметричного) решения задачи обтекания. Важность оценки (4) объясняется тем, что она влечет асимптотическое разложение решения, определяемое фундаментальным решением системы Озеена [8], при этом же предположении установлены асимптотические формулы для $\omega=\operatorname{rot} w$ и $p$ (см. [9]).

Отметим, что в отличие от [7] приводимый ниже вывод соотношения (4) основан на точных $L_{p}$-оценках решения $w$, получаемых из интегрального представления $w$ через гидродинамические потенциалы.

В дальнейшем, не нарушая общности, считаем, что $\nu=1$, a $(w, p)$ - решение задачи обтекания с $|\nabla w| \in L_{2}(\Omega)$, удовлетворяющее предельному соотношению (3) c $w_{\infty}=(1,0)$. Кроме того, будем использовать следующие установленные в [4]-[6] свойства таких решений:

a) $|\nabla p| \in L_{2}(\Omega)$, существует $\lim _{|x| \rightarrow \infty} p(x)$ (далее считаем его всегда равным 0 );

б) для $\omega=\operatorname{rot} w$ справедливы соотношения

$$
|x|^{1 / 2}|\nabla \omega|,|\omega| \in L_{2}(\Omega), \quad|\omega(x)|=o\left(|x|^{-3 / 4}\right), \quad|\nabla w(x)|=o\left(|x|^{-3 / 4} \ln |x|\right) ;
$$

в) функция $\omega$ и функция Бернулли $\Phi=p+|w|^{2} / 2-1 / 2$ экспоненциально убьвают вне любого сектора, содержащего положительную $x_{1}$-полуось: $\omega=o\left(e^{-\alpha|x|}\right)$, $\Phi=o\left(e^{-\alpha|x|}\right)$, где $\alpha>0$ зависит от угла раствора сектора.

2. В дальнейшем существенную роль играют некоторые оценки фундаментального решения $\left(u_{j}^{k}, q^{k}\right), k, j=1,2$, линеаризованной системы Озеена

$$
\nu \Delta u-\partial_{1} u-\nabla q=f, \quad \operatorname{div} u=0,
$$

определяемого формулами

$$
\begin{gathered}
q^{k}(x)=\frac{1}{2 \pi} \partial_{k} \ln \frac{1}{|x|}, \quad u_{1}^{1}(x)=\frac{1}{2}\left(-e^{x_{1} / 2} K_{0}\left(\frac{|x|}{2}\right)+\partial_{1} \Omega(x)\right) \\
u_{2}^{1}(x)=u_{1}^{2}(x)=\frac{1}{2 \pi} \partial_{2} \Omega(x), \quad u_{2}^{2}(x)=-\frac{1}{2 \pi} \partial_{1} \Omega(x)
\end{gathered}
$$

где $\Omega(x)=\ln |x|+e^{x_{1} / 2} K_{0}(|x| / 2), K_{0}(r)$ - функция Макдональда. 
Вследствие асимптотики функции Макдональда $K_{0}(r) \sim e^{-r} / \sqrt{2 \pi r}$ при $r \rightarrow \infty$ справедливы неравенства

$$
\left|u_{j}^{k}(x)\right| \leqslant \begin{cases}c\left(\frac{1}{|x|}+\frac{e^{\left(x_{1}-|x|\right) / 2}}{\sqrt{|x|}}\right), & (k, j)=(1,1), \\ c \frac{1}{|x|}, & (k, j) \neq(1,1),\end{cases}
$$

из которых, в частности, следует, что

$$
u_{j}^{k}(x) \in L_{p}\left(\mathbb{R}^{2}\right)
$$

где $p \in(3, \infty)$ при $(k, j)=(1,1)$ и $p \in(2, \infty)$ при $(k, j) \neq(1,1)$.

Соответствуюший анализ производных фундаментального решения показывает, что

$$
\partial_{s} u_{j}^{k} \in L_{p}\left(\mathbb{R}^{2}\right)
$$

где $p \in(3 / 2,2)$ при $(k, j, s)=(1,1,2)$ и $p \in(1,2)$ при $(k, j, s) \neq(1,1,2)$, причем вне окрестности нуля

$$
\partial_{s} u_{j}^{k} \in L_{p}(|x|>1),
$$

г де $p \in(3 / 2, \infty]$ при $(k, j, s)=(1,1,2)$ и $p \in(1, \infty]$ при $(k, j, s) \neq(1,1,2)$.

Далее, применение теорем о свертке и операторах типа потенциала (см., например, [10]) дает следующие оценки:

$$
\left\|u_{j}^{k} * f\right\|_{L_{q}\left(\mathbb{R}^{2}\right)} \leqslant c_{p, q}\|f\|_{L_{p}\left(\mathbb{R}^{2}\right)},
$$

где $1<p \leqslant q<\infty, 1 / q=1 / p+1 / r-1$, причем $r \in[3, \infty)$ при $(k, j)=(1,1)$ и $r \in[2, \infty)$ при $(k, j) \neq(1,1)$;

$$
\left\|\partial_{s} u_{j}^{k} * f\right\|_{L_{q}\left(\mathbb{R}^{2}\right)} \leqslant c_{p, q}\|f\|_{L_{p}\left(\mathbb{R}^{2}\right)},
$$

где $1<p \leqslant q<\infty, 1 / q=1 / p+1 / r-1$, причем $r \in[3 / 2,2]$ при $(k, j, s)=(1,1,2)$ и $r \in[1,2]$ при $(k, j, s) \neq(1,1,2)$.

3. Доказательство основного результата базируется на следующих леммах.

Лемма 1. Для поля $v=w-w_{\infty}$ имеет место интегральное представление

$$
v_{j}(x)=\int_{\Omega} K_{j i}(x-y, v(y)) v_{i}(y) d y+F_{j}(x),
$$

əде

$$
\begin{gathered}
K_{11}=\left(\partial_{1} u_{1}^{1}\right) v_{1}+\left(\partial_{2} u_{1}^{1}+\partial_{1} u_{2}^{1}\right) v_{2}, \quad K_{12}=\left(\partial_{2} u_{2}^{1}\right) v_{2}, \quad K_{21}=0 \\
K_{22}=\left(\partial_{1} u_{2}^{2}-\partial_{2} u_{1}^{2}\right) v_{1}+\left(\partial_{2} u_{2}^{2}\right) v_{2}+2 u_{1}^{2} \partial_{2} v_{1}, \\
F_{1}(x)=\int_{\Omega}\left(n_{1} u_{1}^{1}-u_{j}^{1} n_{s} \partial_{s} v_{j}+n_{s} \partial_{s} u_{1}^{1}-n_{1} u_{1}^{1}+p n_{j} u_{j}^{1}-n_{1} q^{1}\right) d S_{y}, \\
F_{2}(x)=\int_{\Omega}\left(n_{s} \partial_{s} u_{1}^{2}-u_{j}^{2} n_{s} \partial_{s} v_{j}-n_{1} u_{1}^{2}+n_{j} u_{j}^{2} p-q^{2} n_{1}\right) d S_{y}
\end{gathered}
$$


причем в приведенных формулах по повторяющимся индексам производится суммирование; аргументом функиий $u_{j}^{i}, q^{j}$ является $x-y ; v_{j}=v_{j}(y), p=p(y)$; $n_{j}=n_{j}(y)$ - компоненты внешней нормали на $\partial \Omega$ по отношению $\kappa \Omega$.

Справедливость интегрального представления (11) устанавливается стандартным образом. После подстановки $w=v+w_{\infty}$ система (1) умножается на матрицу $u_{i}^{j}(x-y)$ и интегрируется по усеченной области $\Omega_{R}$, интегралы по области $\Omega_{R}$ преобразуются в интегралы по границе $\partial \Omega_{R}$ с помошю формулы Грина, при последующем предельном переходе при $R \rightarrow \infty$ ввиду оценок (5) криволинейные интегралы по окружности $\{y:|y|=R\}$ исчезают (для этого достаточно вьполнения условий $p(y) \rightarrow 0, v_{j}(y) \rightarrow 0$, $\partial_{s} v_{j}(y) \rightarrow 0$ при $\left.|y| \rightarrow \infty\right)$.

Отметим, что при преобразовании интегралов

$$
\int_{\Omega} u_{j}^{k}(x-y)(v(y), \nabla) v_{j}(y) d y
$$

используются соотношения

$$
(v, \nabla) v_{2}=\partial_{1}\left(v_{1} v_{2}\right)+\partial_{2}\left(v_{2}^{2}\right), \quad(v, \nabla) v_{1}= \begin{cases}\partial_{1}\left(v_{1}^{2}\right)+\partial_{2}\left(v_{1} v_{2}\right), & k=1 \\ -\partial_{2}\left(v_{1} v_{2}\right)+2 v_{2} \partial_{2} v_{1}, & k=2\end{cases}
$$

Применение оценок (9), (10) приводит к следующему результату.

ЛЕмма 2. Для интегральных операторов

$$
\left(A_{j i, \rho} f\right)(x)=\int_{|y|>\rho} K_{j i}(x-y, v(y)) f(y) d y, \quad j, i=1,2, \quad(j, i) \neq(2,1),
$$

справедливы неравенства $\left\|A_{j i, \rho}\right\|_{L_{p}(|x|>\rho) \rightarrow L_{p}(|x|>\rho)} \leqslant c_{j i, \rho}$, где $p \in(2, \infty) u$

$$
\begin{gathered}
c_{11, \rho}=c_{p}\left(\max _{|x|>\rho}|v(x)|+\left\|v_{2}\right\|_{L_{3}(|x|>\rho)}\right), \quad c_{12, \rho}=c_{p} \max _{|x|>\rho}\left|v_{2}(x)\right|, \\
c_{22, \rho}=c_{p}\left(\max _{|x|>\rho}|v(x)|+\left\|\partial_{2} v_{1}\right\|_{L_{2}(|x|>\rho)}\right)
\end{gathered}
$$

причем $c_{p}-$ константы, зависящие лишь от $p$.

ЛЕмма 3. Пусть поле $v=w-w_{\infty}$ принадлежит некоторому пространству $L_{p_{0}}(\Omega), p_{0} \in(3, \infty)$. Тогда справедливы включения $v_{i} \in L_{p}(\Omega)$, где $p \in(3, \infty]$ при $i=1$ u $p \in(2, \infty]$ npu $i=2$.

ДокАЗАТЕЛЬСТво. Остановимся подробно на доказательстве включения для компоненты $v_{2}(x)$. Из интегрального представления (11) при $j=2$ вытекает равенство

$$
v_{2}=A_{22, \rho} v_{2}+F_{2, \rho}, \quad \text { где } F_{2, \rho}=F_{2}+\int_{\Omega \cap\{y:|y|>\rho\}} K_{22} v_{2} d y .
$$

Из оценок фундаментального решения (6)-(8) следует, что $F_{2, \rho} \in L_{p}(|x|>\rho)$ для всех $p \in(2, \infty)$. Пусть $q$ - любое фиксированное число из интервала $(2, \infty)$. Выбирая $\rho$ настолько большим, чтобы вьполнялось неравенство

$$
\left\|A_{22, \rho}\right\|_{L_{p}(|x|>\rho) \rightarrow L_{p}(|x|>\rho)}<1
$$


при $p=q$ и $p=p_{0}$, и рассматривая соотношение (12) как интегральное уравнение относительно $v_{2}(x)$ в пространстве $L_{p_{0}}(|x|>\rho)$, получаем, что при $|x|>\rho$

$$
v_{2}(x)=\sum_{n=0}^{\infty}\left(A_{22, \rho}^{n} F_{2, \rho}\right)(x)
$$

Поэтому ввиду неравенства (13) при $p=q$ функция $v_{2}(x)$ принадлежит пространству $L_{q}(|x|>\rho)$. В силу произвольности $q \in(2, \infty)$ утверждение леммы справедливо для компоненты $v_{2}(x)$.

Далее, соответствующий результат для функции $v_{1}(x)$ получается аналогично предыдущему из представления

$$
v_{1}=A_{11, \rho} v_{1}+F_{1, \rho}, \quad \text { где } \quad F_{1, \rho}=F_{1}+\int_{\Omega} K_{12} v_{2} d y+\int_{\Omega \cap\{y:|y|>\rho\}} K_{11} v_{1} d y
$$

так как согласно оценкам фундаментального решения, установленному выше включению $v_{2} \in L_{p}(\Omega)$ при всех $p \in(2, \infty)$ и лемме 2 функция $F_{1, \rho}$ принадлежит всем пространствам $L_{p}(|x|>\rho)$ при $p \in(3, \infty)$.

Таким образом, ввиду леммы 3 для получения точных $L_{p}$-оценок поля $v$ следует установить какие-нибудь “затравочные” оценки. Получение таких оценок составляет содержание следующего пункта.

4. Рассмотрим функцию Бернулли $\Phi=p+|w|^{2} / 2-1 / 2$, удовлетворяющую уравнению $\Delta \Phi=(w, \nabla) \Phi+\omega^{2}$, и систему краевых задач

$$
\begin{gathered}
\Delta \Phi_{R}=(w, \nabla) \Phi_{R}+\omega^{2}, \\
\left.\Phi_{R}\right|_{\partial \Omega}=\left.\Phi\right|_{\partial \Omega},\left.\quad \Phi_{R}\right|_{|x|=R}=0 .
\end{gathered}
$$

Так как для разности $\Phi-\Phi_{R}$ справедлив принцип максимума, а $\Phi(x) \rightarrow 0$ при $|x| \rightarrow \infty$, то последовательность функций $\Phi_{R}$, продолженных нулем на $\Omega \cap\{x:|x|>R\}$, сходится равномерно на $\Omega$ к $\Phi$ и, в частности, $\left\|\Phi_{R}\right\|_{L_{\infty}(\Omega)}$ равномерно ограничены.

Положим $\Phi_{R}=\zeta_{R}+b$, где $b-$ функция, отличная от 0 лишь в некоторой окрестности $\partial \Omega$ и $\left.b\right|_{\partial \Omega}=\left.\Phi\right|_{\partial \Omega}$. Умножая уравнение $(14)$ на $f \zeta_{R}$ и интегрируя по $\Omega_{R}=\Omega \cap\{x$ : $|x|<R\}$, приходим к тождеству

$$
\begin{aligned}
& \int_{\Omega_{R}}\left(\left(\nabla \zeta_{R}\right)^{2} f-\frac{1}{2} \zeta_{R}^{2} \partial_{1} f\right) d x \\
& \quad=-\int_{\Omega_{R}}\left(\zeta_{R}\left(\nabla \zeta_{R}, \nabla f\right)-\frac{1}{2} \zeta_{R}^{2}(v, \nabla) f-f \zeta_{R}\left(\Delta b-(w, \nabla) b-\omega^{2}\right)\right) d x
\end{aligned}
$$

Выбирая

$$
f= \begin{cases}\frac{1}{\ln a}, & x_{1}<a \\ \frac{1}{\ln x_{1}}, & x_{1}>a\end{cases}
$$


из (15) получаем, что при достаточно большом $a>0$ справедлива равномерная по $R$ оценка

$$
\int_{\Omega_{R}} \Phi_{R}^{2}\left|\partial_{1} f\right| d x \leqslant c
$$

(Мы использовали, что $\omega \in L_{2}(\Omega), v \rightarrow 0$ при $|x| \rightarrow \infty$, а функции $\zeta_{R}$ равномерно ограничены.) Переходя к пределу при $R \rightarrow \infty$ и используя экспоненциальное убьвание $\Phi$ вне полуоси $x_{1}>0$, устанавливаем, что

$$
\int_{\Omega} \Phi^{2}(x) \frac{d x}{|x| \ln ^{2}|x|} \leqslant c .
$$

Далее, справедлива

ЛЕмма 4. Для поля $v$ при любом $\varepsilon>0$ выполнена оценка

$$
\int_{\Omega} v^{2} \frac{d x}{|x|^{1+\varepsilon}}<\infty
$$

ДокАЗАТЕЛЬСТво. Для вьвода (17) установим интегральную формулу, связьвающую поле $v$ с функцией Бернулли $\Phi$. Введем комплексную скорость $u=w_{1}+i w_{2}$ и дифференциальные операторы

$$
\frac{\partial}{\partial \bar{z}}=\frac{1}{2}\left(\frac{\partial}{\partial x_{1}}+i \frac{\partial}{\partial x_{2}}\right), \quad \frac{\partial}{\partial z}=\frac{1}{2}\left(\frac{\partial}{\partial x_{1}}-i \frac{\partial}{\partial x_{2}}\right) .
$$

В этих терминах систему Навье-Стокса можно представить в форме

$$
4 \frac{\partial^{2} u}{\partial \bar{z} \partial z}-2 \frac{\partial}{\partial \bar{z}} \Phi=\frac{\partial}{\partial z}\left(u^{2}-1\right) .
$$

Умножая (18) на функцию $1 /(\pi(\bar{z}-\bar{w}))$, интегрируя по области

$$
\Omega_{\varepsilon, R}=(\Omega \backslash\{z:|z-w|<\varepsilon\}) \cap\{z:|z|<R\}
$$

и переходя затем к пределу при $\varepsilon \rightarrow 0, R \rightarrow \infty$, получаем формулу

$$
u^{2}(w)-1=(S \Phi)(w)+f(w)
$$

где $S$ - сингулярный оператор

$$
\begin{gathered}
(S \Phi)(w)=\frac{2}{\pi} \int_{\Omega} \frac{\Phi(z)}{(\bar{z}-\bar{w})^{2}} d x_{1} d x_{2} \\
f(w)=4 \frac{\partial u}{\partial \bar{z}}(w)-\frac{1}{\pi i} \int_{\partial \Omega} \frac{\Phi}{\bar{z}-\bar{w}} d z+\frac{1}{2 \pi i} \int_{\partial \Omega} \frac{u^{2}-1-4 \partial u / \partial \bar{z}}{\bar{z}-\bar{w}} d \bar{z}
\end{gathered}
$$

Ввиду того что $\partial u / \partial \bar{z} \in L_{2}(\Omega)$, функция $f$ принадлежит любому весовому пространству $L_{2}\left(\Omega,|x|^{-\alpha}\right)$ при любом $\alpha>0$. Из оценки (16) следует, что $\Phi \in L_{2}\left(\Omega,|x|^{-1-\varepsilon}\right)$ для сколь угодно малого $\varepsilon>0$. В силу ограниченности сингулярного интеграла $S$ в любом весовом пространстве $L_{2}\left(\Omega,|x|^{-\alpha}\right), 0 \leqslant \alpha<2$, из (19) получаем, что $u^{2}-1 \in L_{2}(\Omega$, $\left.|x|^{-1-\varepsilon}\right)$ при любом $\varepsilon>0$. Из равенства $u^{2}-1=w_{1}^{2}-1-w_{2}^{2}+2 i w_{1} w_{2}$, поскольку $w_{1} \rightarrow 1, w_{2} \rightarrow 0$ при $|x| \rightarrow \infty$, получаем $w_{2}, w_{1}-1 \in L_{2}\left(\Omega,|x|^{-1-\varepsilon}\right)$.

Таким образом, лемма 4 установлена. 
5. В этом пункте завершается доказательство оценки (4).

ТЕОрема 1. Для решения задачи обтекания справедлива оченка (4).

ДокАЗАТЕльство. Для $\omega=\operatorname{rot} w$ и поля $v=w-w_{\infty}$ справедливо соотношение

$$
\Delta v=\nabla^{\perp} \omega
$$

где $\nabla^{\perp}=\left(-\partial_{2}, \partial_{1}\right)$.

Пусть $x \in \Omega$ и $B_{\rho}=\{y:|x-y|<\rho\}-$ шар с центром в $x$, содержашийся в области $\Omega$. Умножая равенство $(20)$ на функцию $(2 \pi)^{-1} \ln (|x-y| / \rho)$ и интегрируя по шару $B_{\rho}$, после известных преобразований получаем формулу

$$
v(x)=\frac{1}{2 \pi} \int_{\partial B_{\rho}} \frac{v(y)}{\rho} d s+\frac{1}{2 \pi} \int_{B_{\rho}} \frac{\omega(y)(x-y)^{\perp}}{(x-y)^{2}} d y
$$

где $(x-y)^{\perp}=\left(-\left(x_{2}-y_{2}\right), x_{1}-y_{1}\right)$.

Интегрируя по $\rho$ от $R / 2$ до $R$ и оценивая полученные интегралы, имеем

$$
|v(x)| \leqslant c\left(\int_{B_{R}} \frac{|v(y)|}{R^{2}} d y+\int_{B_{R}} \frac{|\omega(y)|}{|x-y|} d y\right)
$$

Учитьвая, что $v \in L_{2}\left(\Omega,|x|^{-1-\varepsilon}\right)$, из (21) получаем оценку

$$
|v(x)| \leqslant c R^{-2}\left(\int_{\Omega} \frac{|v(y)|^{2}}{|y|^{1+\varepsilon}} d y\right)^{1 / 2}\left(\int_{B_{R}}|y|^{1+\varepsilon} d y\right)^{1 / 2}+c \max _{y \in B_{R}}|\omega(y)| R .
$$

Считая $|x|$ достаточно большим, а $R<|x| / 2$, устанавливаем неравенство

$$
v(x) \leqslant c\left(|x|^{(1+\varepsilon) / 2} R^{-1}+|x|^{-3 / 4} R\right) .
$$

Минимизируя его по $R$, получаем, что $|v(x)| \leqslant c|x|^{(-1+2 \varepsilon) / 8}$. Таким образом, $v \in L_{p}(\Omega)$ для всех $p>16$. Это дает “затравочные” оценки для применения леммы 3. Поэтому $v \in L_{p}(\Omega)$ при всех $p>3$. Учитывая этот факт, из $(21)$ выводим неравенство

$$
|v(x)| \leqslant c\left(R^{-2 / p}\left(\int_{B_{R}}|v|^{p} d x\right)^{1 / p}+R|x|^{-3 / 4}\right) .
$$

Полагая $R=|x|^{\alpha}$ при $\alpha=3 /(4+8 / p)$ и выбирая $p>3$ сколь угодно близким к 3 , получаем $|v(x)| \leqslant c|x|^{-3 / 10+\varepsilon}$, что и завершает доказательство. 


\section{СПИСОК ЦИТИРОВАННОЙ ЛИТЕРАТУРЫ}

[1] Leray J. Étude de diverses équations intégrales non linéaires et de quelques problèmes que pose l'hydrodynamique // J. Math. Pures Appl. 1933. V. 12. P. 1-82.

[2] Ладыженская О. А. Математические вопросы динамики вязкой несжимаемой жидкости. М.: Наука, 1970.

[3] Finn R., Smith D. R. On the stationary solution of the Navier-Stokes equations in two dimensions // Arch. Rational Mech. Anal. 1967. V. 25. P. 26-39.

[4] Вайнбергер Г.Ф., Гилбарг Д. Асимптотические свойства решений Лере стационарных двумерных уравнений Навье-Стокса // УМН. 1974. Т. 29. № 2. С. 109-122.

[5] Gilbarg D., Weinberger H.F. Asymptotic properties of steady plane solutions of the Navier-Stokes equations with bounded Dirichlet integral // Ann. Scuola Norm. Sup. Pisa. Cl. Sci. (4). 1978. V. 5. P. 381-404.

[6] Amick C. J. On Leray's problem of steady Navier-Stokes flow past a body in the plane // Acta Math. 1988. V. 161. P. 71-130.

[7] Amick C. J. On the asymptotic form of Navier-Stokes flow past a body in the plane // J. Differential Equations. 1991. V. 1. P. 149-167.

[8] Smith D. R. Estimates at infinity for stationary solutions of Navier-Stokes equations // Arch. Rational Mech. Anal. 1965. V. 20. P. 341-372.

[9] Бабенко К.И. Об асимптотическом поведении вихря вдали от тела при обтекании его потоком вязкой жидкости // Прикладная матем. и механика. 1970. Т. 34. С. 911-925.

[10] Стейн И. М. Сингулярные интегралы и дифференциальные свойства функций. М.: Мир, 1973.

Ростовский государственный университет

Поступило 02.04 .97

Исправленный вариант

01.06 .98 